\title{
Orofacial Clefts in High Prevalence Area of Birth Defects - Five Counties, Shanxi Province, China, 2000-2020
}

\author{
Jufen Liu ${ }^{1,2} ;$ Yali Zhang ${ }^{1,2}$; Le Zhang ${ }^{1,2} ;$ Linlin Wang ${ }^{1,2} ;$ Lei Jin $^{1,2} ;$ Nicholas D E Greene ${ }^{3} ; Z_{\text {Zhiwen Li }}^{1,2, * ;}$; Aiguo Ren ${ }^{1,2}$
}

\section{Summary \\ What is already known on this topic? \\ The prevalence of structural birth defects, especially neural tube defects, decreased after national folic acid (FA) supplementation initiation. \\ What is added by this report? \\ The prevalence of orofacial clefts (OFCs) in five counties of Shanxi Province in northern China, including most subtypes except cleft palate, showed a downward trend in the past two decades. In this study, pre-perinatal prevalence increased due to earlier detection. \\ What are the implications for public health practice? \\ Periconceptional supplementation with FA may contribute to the decline in OFCs prevalence, while the effect on the OFCs subtype needs further investigation. Continuing to advocate for earlier supplementation (3 months before conception) and increased supplementation frequency (daily consumption) could promote further reduction in the prevalence of OFCs. Specific surveillance of this effect in the era of universal three-child policy is warranted.}

Orofacial clefts (OFCs) are among the most common human congenital malformations worldwide, and the majority of OFCs are non-syndromic (1). Although most OFCs are not fatal, children born with non-syndromic cleft lip, with or without cleft palate (NSCL/P), may have low intelligence and/or impaired speech/language development (2). In addition, OFCs also impose significant social, financial, and public health burdens. Periconceptional folic acid (FA) supplementation could help reduce the risk of neural tube defects (NTDs) (3) and other selected structural birth defects, including OFCs (4). However, the effect on different subtypes of OFCs has not been thoroughly evaluated. Similarly, the effect of policy changes, including the change from mandatory pre-marital health examination to voluntary examinations, release of the two-child population policy, and the subsequent update to the ongoing three-child policy, have not yet been explored. The current study examined the trend of OFCs in 5 counties based on data from a population-based birth-defect surveillance system in a high prevalence area of northern China from 2000 to 2020. The prevalence of OFCs in the 5 counties in Shanxi Province decreased significantly in the past two decades. Periconceptional supplementation with FA may have contributed to the decline in OFC prevalence.

The data used in the current study came from a population-based birth-defect surveillance system in Shanxi Province. Details of the system have been described in our previous publication (3). In summary, all livebirths or stillbirths of 28 or more complete gestational weeks and pregnancy terminations at any gestational age following the prenatal diagnosis of birth defects among pregnant women who reside in five counties located in Shanxi Province (Pingding, Shouyang, Taigu, Xiyang, and Zezhou) for more than 1 year were included. OFCs were coded Q35-37 according to the International Statistical Classification of Diseases and Related Health Problems, 10th revision (ICD-10) as different types of birth defect collected in the system (Table 1). The birth prevalence of OFCs by year, period, type, and gestational week's group was compared using chi-squared tests. Twotailed $P \leq 0.05$ was considered statistically significant. All statistical analyses were performed using SPSS Statistics for Windows (Version 24.0. IBM Corp., Armonk, NY, USA).

From 2000 to 2020 , a total of 302,101 births and 712 cases of OFCs were recorded in the system, resulting in a total prevalence of $23.57 / 10,000$ births. The proportion of perinatal OFCs with $\geq 28$ gestational weeks accounted for $100 \%$ in 2000 and $41.7 \%$ in 2020 (Figure 1A) while the proportion of pre-perinatal OFCs with $<28$ gestational weeks accounted for $0 \%$ in 2000 and $58.3 \%$ in 2020. The perinatal prevalence decreased dramatically, from more than 30/10,000 in 2002 to merely 5.9/10,000 in 2020 (chi-squared: $39.922, \quad P<0.05) \quad$ (Figure 1B). The 
TABLE 1. Prevalence of orofacial clefts (OFCs) by subtype and gestational weeks in 5 counties of Shanxi Province, 2000-2020.

\begin{tabular}{|c|c|c|c|c|c|c|}
\hline \multirow{3}{*}{ ICD-10 code } & \multicolumn{6}{|c|}{ Gestational weeks } \\
\hline & \multicolumn{2}{|r|}{$<28$ weeks } & \multicolumn{2}{|r|}{$\geq 28$ weeks } & \multicolumn{2}{|r|}{ Total } \\
\hline & $\mathbf{n}$ & Prevalence, $1 / 10,000$ & $\mathbf{n}$ & Prevalence, $1 / 10,000$ & $\mathbf{n}$ & Prevalence, $1 / 10,000$ \\
\hline Cleft palate (Q35) & 4 & 0.13 & 98 & 3.24 & 102 & 3.38 \\
\hline Cleft lip (Q36) & 38 & 1.26 & 249 & 8.24 & 287 & 9.50 \\
\hline Cleft lip with cleft palate (Q37) & 48 & 1.59 & 275 & 9.10 & 323 & 10.69 \\
\hline Total & 90 & 2.98 & 622 & 20.59 & 712 & 23.57 \\
\hline
\end{tabular}
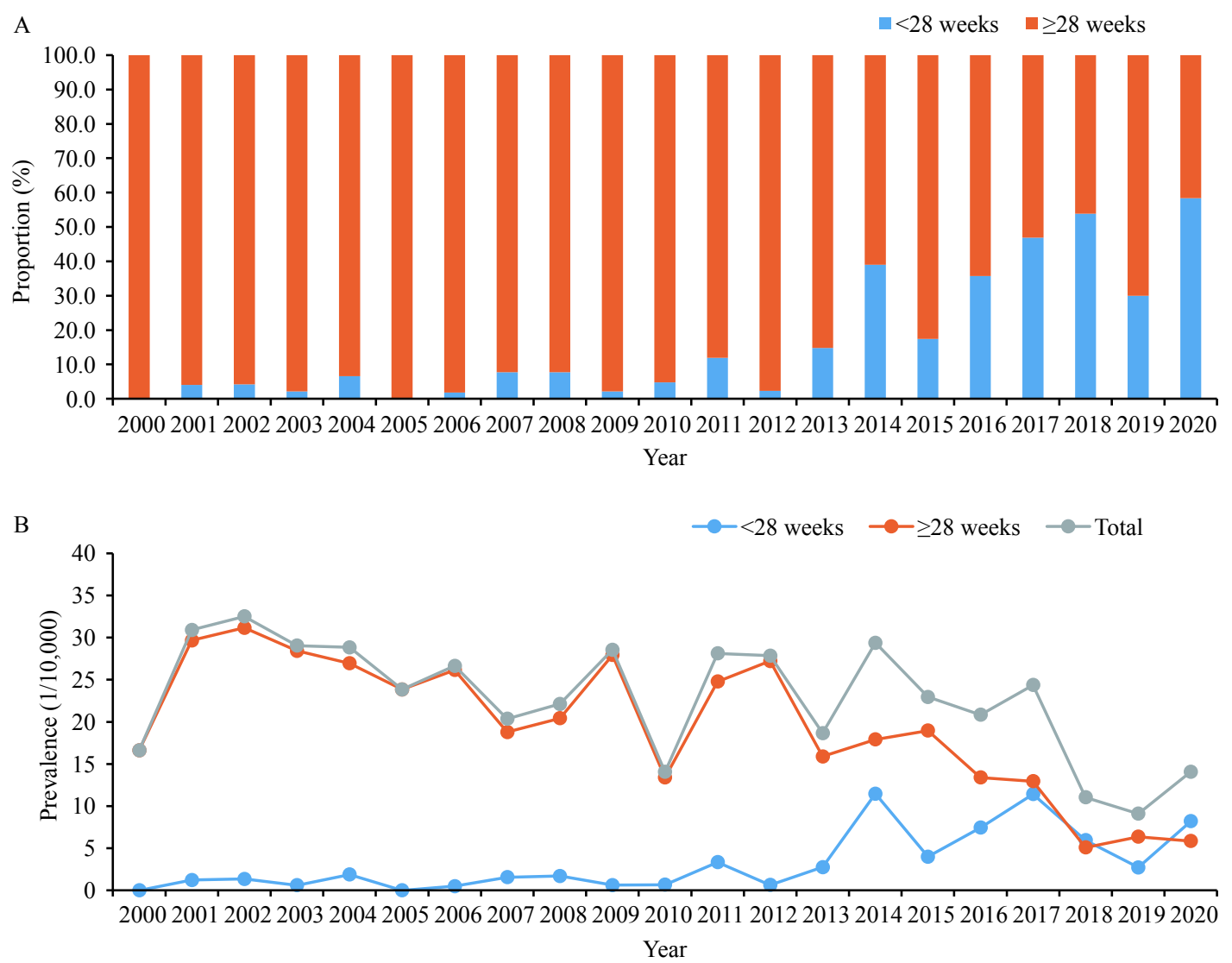

FIGURE 1. Orofacial clefts (OFCs) by gestational week and year in 5 counties, Shanxi Province, China, 2000-2020. (A) Proportion of OFCs; (B) Prevalence of OFCs.

Note: Perinatal prevalence (cases of 28 or more gestational weeks), pre-perinatal prevalence (cases before 28 gestational weeks), and total prevalence (all cases regardless of gestational age) were calculated. Significant time points and corresponding population policy and public strategies are as follows: 2003 , canceled mandatory pre-marital physical checkups; 2009, national campaign for FA supplementation; 2012, population policy transition; and 2016, release of universal twochild policy.

decreasing trend in overall OFCs was also reflected in decreasing frequency of OFCs detected at perinatal stage (Figure 2A) (chi-squared: 120.001, $P<0.05$ ). While the pre-perinatal OFCs showed an upward trend during the past two decades due to early detection.

Cleft lip with cleft palate was the most common type among all OFCs, followed by cleft lip alone, and isolated cleft palate was the third most common type. The rate of cleft lip with cleft palate was 10.69 per 10,000 births and accounted for $45 \%$ of OFCs in this population. The prevalence of OFCs detected at the perinatal stage $(20.59 / 10,000)$ was higher than that detected at pre-perinatal stage $(2.98 / 10,000)$ (Table 1$)$.

Among all types of OFCs, the prevalence of cleft lip decreased the most (Figure 2B): from 15.15 per 10,000 


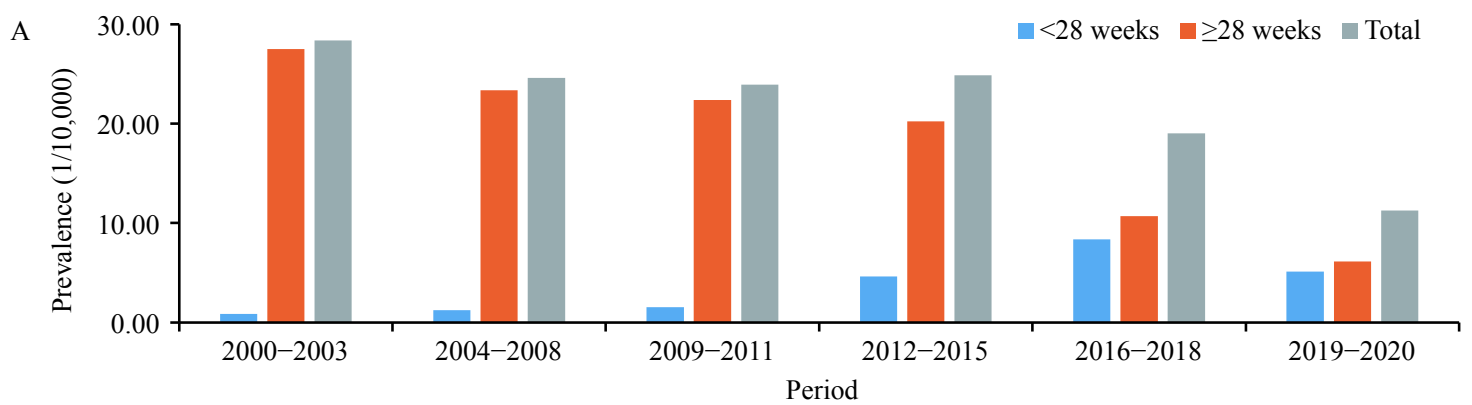

B

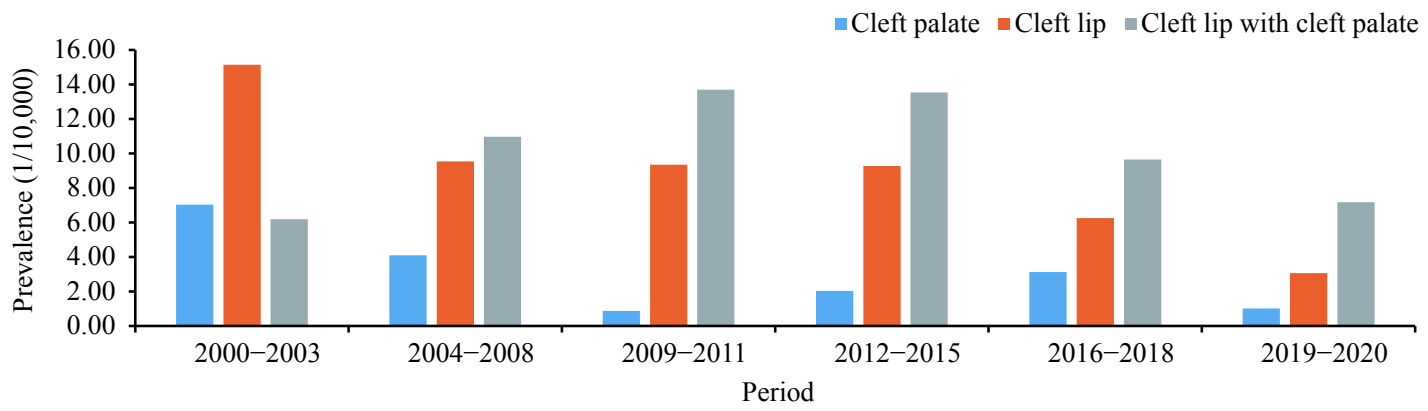

FIGURE 2. Prevalence of orofacial clefts (OFCs) by period in 5 counties of Shanxi Province, 2000-2020. (A) pooled OFCs by gestational weeks; (B) OFCs by subtype.

Note: Perinatal prevalence (cases of 28 or more gestational weeks), pre-perinatal prevalence (cases before 28 gestational weeks), and total prevalence (all cases regardless of gestational age) were calculated. The 6 periods were divided according to population policy and public strategy, i.e., 2000-2003 (Period before voluntary pre-marital physical check-ups), 2004-2008 (Period of voluntary pre-marital physical check-ups and before FA supplementation), 2009-2011 (Period of after FA supplementation), 2012-2015 (Period of population policy transition), 2016-2018 (Period of universal two-child policy), and 2019-2020.

live births between 2000-2003 to 9.35 per 10,000 live births between 2009-2011; from 6.26 per 10,000 live births between 2016-2018 to 3.08 per 10,000 live births between 2019-2020; an overall reduction of $80 \%$ in the past 2 decades (Overall reduction, chisquared: $28.526, P<0.05)$. Cleft lip with cleft palate increased from 6.19 per 10,000 live births to as high as 13.70 per 10,000 live births between 2000-2011 and decreased to 7.18 per 10,000 live births between 2019-2020 (Overall reduction, chi-squared: 21.356, $P<0.05)$. Cleft palate showed a decreasing trend from 7.04 to 0.87 per 10,000 live births during the first decade and slightly increased to $2-3$ per 10,000 live births after that (Overall reduction, chi-squared: 34.837, $P<0.05)$.

\section{DISCUSSION}

This population-based birth defect surveillance system showed that the overall prevalence of OFCs in the 5 counties in Shanxi Province decreased significantly from 30.9 per 10,000 births to 14.1 per 10,000 in the past two decades. We hypothesize that the introduction of periconceptional supplementation with FA may have contributed to this decline. In this case, continuing to advocate for earlier supplementation with FA, which should start from at least 3 months before conception, and increased frequency of supplementation (daily consumption) may promote further reduction of OFCs. The overall prevalence of OFCs showed a continuous decreasing trend when live births significantly declined in China. Specifically monitoring the prevalence of OFCs in the era of the universal three-child policy is warranted.

The prevalence of OFCs in these 5 counties of Shanxi Province was still high, with 14.1 per 10,000 births in 2020, which was significantly higher than the national average and Guangdong Province (7.55 per 10,000 births), Cuba (7.8 per 10,000 births), and the United States (10.0 per 10,000 births). Risk factors for OFCs have been extensively explored. Genetic mutations that disrupt folate metabolism and transportation pathways, including methylenetetrahydrofolate reductase (MTHFR), and reduced folate carrier (RFC1) were studied among the Chinese population (5). A moderate association between MTHFR C677T polymorphism with NSCL/P was revealed in a population from northern 
China, and there was a higher incidence associated with the $\mathrm{T}$ allele in the north than in the south (G). Future studies of the genetic basis of OFCs are required among northern Chinese populations.

OFCs have a complex etiology resulting from genetic variants combined with environmental exposure factors. Socioeconomic improvements, including higher earnings and higher education levels, would likely better prevent birth defects. Environmental factors including nutrition, medication use, and chemical exposures could also influence the risk for OFCs. FA fortification may have beneficial effects on non-syndromic OFCs $(\mathrm{RR}=0.88 ; 95 \% \mathrm{CI}$ : $0.81-0.96$ ) (7). Our study revealed a continuous decline in the prevalence of OFCs after the initiation of comprehensive FA supplementation among Chinese pregnant women, similar to the trend observed for neural tube defects (4). The nationwide FA supplementation program started from rural areas since 2009; our study showed that the prevalence of OFCs after 2009 was significantly lower than that before 2009, especially cleft lip with/without cleft palate. Therefore, women residing in the 5 counties in this study may have benefited from the program providing free FA supplements. The effect of OFCs on cleft palate requires further investigations as suggested by a recent case-control study (8). As FA supplementation has a strong impact on plasma folate concentrations, earlier supplementation and increased supplementation frequency could potentially promote further reduction of birth defects (9). Our previous study revealed that in utero exposure to $\mathrm{As}, \mathrm{Cd}, \mathrm{Pb}$, and $\mathrm{Ni}$ might increase the risks of OFCs in newborns, and a dose-response relationship between risks for total OFCs and different types of OFCs, and the aforementioned heavy metals was observed (10). A further two-stage study revealed that exposure to $\mathrm{Pb}$ increased the risk for NSCL/P and this may partly be explained by hypermethylation of WNT3A (11). Besides DNA methylation, other epigenetic modifications, such as histone modifications and microRNA expressions, are emerging mechanisms of importance for orofacial development.

Cleft lip with or without cleft palate decreased significantly during the past two decades. In our study, although the total prevalence of OFCs decreased with time, pre-perinatal OFCs increased. The improvement of the detection method could have contributed to this increase. With the implementation of FA supplementation, more women understood the importance of the pre-natal screening program and went to have prenatal checks earlier. Modern ultrasound technology assists obstetricians and gynecologists to identify structural malformations such that the proportion of OFCs discovered before 28 weeks increased. Early prenatal examination still needs to be applied more widely in the future.

The number of live births in the study areas have declined since 2016, especially between 2019-2020, which coincided with a decreased fertility rate and may be related to the changing population structure, so further studies are still needed to explore the possible effect of population policy changes as well as fertility transitions. The total number of live births was as high as 20,000 around 2004-2006 but only 8,528 in 2020 . As advanced maternal age (AMA) is associated with an overall increased risk for major anomalies, following the enactment of the universal three-child policy in 2021, the risk for OFCs due to AMA may deserve more attention in the near future.

The strength of the current study resides in the population-based surveillance data which included all birth defects regardless of gestational weeks. Our study could provide a more accurate estimation than that provided by hospital-based surveillance data (which only included birth defects detected at more than 28 gestational weeks). Secondly, we collected data over a period of more than 20 years which enabled the analysis of long-term trends.

This study was subject to some limitations. There was a lack of detailed information on the details of NSCL/P, for example whether cleft was bilateral or unilateral, located in hard or soft palate. The study areas were located in Shanxi Province and only limited counties were included, which only reflected the situation of high prevalence of birth defects. Furthermore, no gender effect was considered.

Acknowledgements: Collaborating hospital staff from Pingding, Shouyang, Taigu, Xiyang, and Zezhou counties.

Funding: This study is supported by the National Key Research and Development Program, Ministry of Science and Technology from P.R. China (Grant No. 2016YFC1000501), Natural Science Foundation of China (No.81973056) and Medical Research Council (Grant Ref: MR/T003847/1) from UK.

doi: $10.46234 / \mathrm{ccdcw} 2021.192$

\# Corresponding author: Zhiwen Li, lizw@bjmu.edu.cn.

1 Institute of Reproductive and Child Health/Key Laboratory of
Reproductive Health, National Health Commission of the People's
Republic of China, Peking University, Beijing, China; ${ }^{2}$ Department of
Epidemiology and Biostatistics, School of Public Health, Peking
University, Beijing, China; ${ }^{3}$ UCL Great Ormond Street Institute of 
Child Health, University College London, London, UK.

Submitted: August 08, 2021; Accepted: September 01, 2021

\section{REFERENCES}

1. Mossey PA, Little J, Munger RG, Dixon MJ, Shaw WC. Cleft lip and palate. Lancet 2009;374(9703):1773 - 85. http://dx.doi.org/10.1016/ S0140-6736(09)60695-4.

2. Wehby GL, Collet B, Barron S, Romitti PA, Ansley TN, Speltz M. Academic achievement of children and adolescents with oral clefts. Pediatrics 2014;133(5):785 - 92. http://dx.doi.org/10.1542/peds.20133072.

3. Liu JF, Zhang L, Li ZW, Jin L, Zhang YL, Ye RW, et al. Prevalence and trend of neural tube defects in five counties in Shanxi province of northern China, 2000 to 2014. Birth Defects Res A Clin Mol Teratol 2016;106(4):267 - 74. http://dx.doi.org/10.1002/bdra.23486.

4. Liu JF, Wang LL, Zhang YL, Zhang L, Jin L, Li ZW, et al. Selected structural birth defects-Shanxi Province, China, 2000-2019. China CDC Wkly 2020;2(37):718 - 22. http://dx.doi.org/10.46234/ccdcw 2020.196.

5. Pei LJ, Zhu HP, Zhu JH, Ren AG, Finnell RH, Li Z. Genetic variation of infant reduced folate carrier (A80G) and risk of orofacial defects and congenital heart defects in China. Ann Epidemiol 2006;16(5):352 - 6 . http://dx.doi.org/10.1016/j.annepidem.2005.02.014

6. Zhu JH, Ren AG, Hao L, Pei LJ, Liu JM, Zhu HP, et al. Variable contribution of the MTHFR C677T polymorphism to non-syndromic cleft lip and palate risk in China. Am J Med Genet A 2006;140A (6):551 - 7. http://dx.doi.org/10.1002/ajmg.a.31115.

7. Millacura N, Pardo R, Cifuentes L, Suazo J. Effects of folic acid fortification on orofacial clefts prevalence: a meta-analysis. Public Health Nutr 2017;20(12):2260 - 8. http://dx.doi.org/10.1017/ S1368980017000878.

8. Xu WL, Yi L, Deng CF, Zhao ZL, Ran LR, Ren ZH, et al. Maternal periconceptional folic acid supplementation reduced risks of nonsyndromic oral clefts in offspring. Sci Rep 2021;11(1):12316. http://dx.doi.org/10.1038/s41598-021-91825-9.

9. Zhang XJ, Liu JF, Jin YS, Yang S, Song ZJ, Jin L, et al. Folate of pregnant women after a nationwide folic acid supplementation in China. Matern Child Nutr 2019;15(4):e12828. http://dx.doi.org/10. $1111 / \mathrm{mcn} .12828$.

10. Ni WL, Yang WL, Yu JH, Li ZW, Jin L, Liu JF, et al. Umbilical cord concentrations of selected heavy metals and risk for orofacial clefts. Environ Sci Technol 2018;52(18):10787 - 95. http://dx.doi.org/10. 1021/acs.est.8b02404.

11. Yang WL, Guo YN, Ni WL, Tian T, Jin L, Liu JF, et al. Hypermethylation of WNT3A gene and non-syndromic cleft lip and/or palate in association with in utero exposure to lead: a mediation analysis. Ecotoxicol Environ Saf 2021;208:111415. http://dx.doi.org/ 10.1016/j.ecoenv.2020.111415. 